\title{
Outcome of Percutaneous pining among Children with displaced Supracondylar Fracture of the Humerus
}

Muhammad Ali, ${ }^{1}$ Kashif Siddiq, ${ }^{1}$ Muhammad Khizer Hayat Makki, ${ }^{1}$ Muhammad Safdar Baig, ${ }^{2}$ Ghulam Qadir Khan, ${ }^{1}$ Shahid Riaz ${ }^{1}$

\begin{abstract}
Background: Percutaneous pining among children with displaced supracondylar fracture of the humerus, may offer additional benefits to patients in terms of outcome.

Objective: To determine the union of fracture and range of motion at elbow, in supracondylar fracture, among children, with closed reduction and percutaneous pinning.

Methodology: A cross-sectional study was conducted, from $19^{\text {th }}$ December 2016 to $19^{\text {th }}$ May 2018, at Ortho Unit 2, Nishtar Hospital, Multan, patients of both sex, having supracondylar fracture (Gartland type II and III) diagnosed radiographically, and presenting within $1^{\text {st }}$ week of injury, were included, and patients with a history of previous surgery or trauma over the arm, compound fracture and neurovascular compromise, were excluded. All patients were operated for the fracture (closed reduction and pinning under image intensifier). The patients were discharged the next day after the procedure. The follow up completed at 6 weeks postoperatively by Flynn's criteria.

Results: Out of 254, 202 (79.5\%) patients were fulfilling the satisfactory level of outcome, $52(20.5 \%)$ patient showed an unsatisfactory level of outcome according to operational definition In this study, 96 (37.8\%) males and 106 (41.2\%) females patients shows satisfactory outcome whereas $23(9.1 \%)$ males and $29(11.4 \%)$ females patients showed unsatisfactory level of outcome $(\mathrm{p}=0.04)$. Out of 254 patients $59(23.2 \%)$ showed excellent, $94(37 \%)$ good, 49 (19.3\%) fair and 52 (20.4\%) poor outcome based on Flynn's criteria.

Conclusion: From our study, the percutaneous pinning of displaced supracondylar fracture of humerus among children under image intensifier is the effective method of treatment in our study setting.

Key words: Supracondylar fractures, Humerus, Percutaneous pinning

Article Citation: Ali M, Siddiq K, Makki MKH, Baig MS, Khan GQ, Riaz S. Outcome of Percutaneous pining among Children with displaced Supracondylar Fracture of the Humerus JSZMC 2020;11(2):29-33. DOI: https://doi.org/10.47883/jszmc.v11i2.24
\end{abstract}

\section{Introduction}

Supracondylar fractures of humerus constitute 3 $\%$ of all the fractures among children and $60 \%$ $70 \%$, of all the fractures around the elbow. Under ten years the supracondylar fractures are common because of different causes mainly ligamentous laxity and anatomical variations of humerus shaft which has thin distal metaphyseal flare which when forced into hyperextension, breaks due to the fulcrum effect of the olecranon. ${ }^{2}$ This thin flare usually fractures at the level of the olecranon fossa. They are generally divided into a Flexion type and an Extension type, second being the commonest and constitute $96 \%$ of the fractures. Gartland further classified it into 3 (three) types depending on the displacement of the distal piece. ${ }^{3}$ Gartland classification of supracondylar fracture of humerus in children includes; type 1, minimally displaced fracture, type 2 , displaced with one cortex intact (posterior), and type 3, completely displaced with both cortices fractured; 3a: posterolateral, and 3b: posteromedial. ${ }^{4}$

The patients usually present with pain, swelling, and inability to move the elbow joint after a fall on the outstretched hand resulting in hyperextension at the elbow. The flexion type usually occurs by a direct injury to the posterior aspect of elbow. ${ }^{2}$ The clinical diagnosis of a displaced supracondylar fracture in children is so obvious that sometimes the neurovascular examination is missed. It is of utmost importance to examine the neurological and vascular status of the limb. These fractures in children are challenging to treat, due to the tendency of loss of reduction, compartment syndrome, and elbow stiffness, neurovascular complications. Treatment by splinting has been noncontroversial for non displaced fractures, but management for displaced

1. Department of Orthopedic Surgery, Civil Hospital Bahawalpur/Quaid-e-Azam Medical College, Bahawalpur, Pakistan.

2. Department of Oral \& Dental Surgery, QMC/BVH/Civil Hospital Bahawalpur, Pakistan.

Correspondence: Dr. Muhammad Ali, Senior Registrar, Department of Orthopedic Surgery, Civil Hospital Bahawalpur/ Quaid-e-Azam Medical College, Bahawalpur. 
fractures is still debatable.

There is more than one correct method suggested for displaced fractures. However, none of them is taken as a gold standard. These include manipulative reduction splinting in a cast, skin or skeletal traction, percutaneous pining under image control, and fixation after open reduction. ${ }^{4}$ Of these, open reduction with internal fixation is the most popular. However, this is associated with possibilities of complications like the stiffness of elbow, varus angular mal-alignment, neurological and vascular damage, and compartment syndrome and myositis ossificans. ${ }^{5,6}$

Percutaneous pinning under image intensifier control is another method that has fewer complications than open reduction. It has the advantage of less surgery time and early effective physical therapy. ${ }^{7,8}$ Two-pin insertion techniques are known: a lateral pining method and crossed pin insertion method. Both methods have their pros and cons in terms of biomechanical stabilization and complications. Gartland type II and III were treated with closed reduction and pinning using image intensifier, and final results were evaluated with Flynn's criteria, with a satisfactory outcome. ${ }^{6,9}$ Recently with the availability of image intensifiers, we started closed reduction percutaneous wiring of supracondylar fracture in children under image control, and the outcome was evaluated.

\section{Methodology}

This cross-sectional study was conducted during the period from 19th December 2016 to 19 May 2018, on 254 patients ranging from 5 years to 12 years of age, of either sex, coming to the emergency department of Ortho Unit, Nishtar Hospital, Multan; with the diagnosis of supracondylar fracture (Gartland type II and III) diagnosed on X-rays, presented within $1^{\text {st }}$ week of injury, were included, and patients with a history of previous surgery or trauma over the arm, compound fracture and neurovascular compromise were excluded. All patients operated by closed reduction method with percutaneous pinning for the fracture. The surgery was performed by an orthopedic surgeon who has at least 5 years of post-fellowship experience after indoor admission, under general anesthesia with the help of an image intensifier.

Under general anesthesia, the child's arm was placed over a radiolucent table, to assess the reduction in the image intensifier. An assistant gave steady traction with the arm in extension, while the surgeon corrected the coronal displacement. Maintaining the traction with the non-dominant hand, the surgeon applied the posterior force to the proximal fragment with fingers if the dominant hand and through the thumb on the olecranon process applies the anterior force. Maintaining these forces the elbow was flexed by the non-dominant hand and was pronated. Keeping the elbow flexed, displacement was checked on Anteroposterior and Lateral views through an image intensifier.

The percutaneous pinning was done using two smooth k wires (size of the wire is dependent on the age of patient and width of bone) in cross wire fixation procedure. The fracture was maintained in the reduced position by the surgeon non- dominant hand. The lateral pin was inserted first from distal proximally through the center of the lateral condyle and placement was confirmed by the image intensifier.

Then the medial pin was inserted from proximal to distally entering a few degrees dorsal to the midcoronal plane. It ensured that the pin did not cross the medial epicondyle. The final fracture and pins assessment was done on AP and Lateral views under image intensifier. The wires were cut outside the skin after bending. The arm was immobilized in 90 degrees of elbow flexion with a posterior splint.

The patients were discharged the next day after the procedure. The posterior splint was removed at 2 weeks to allow active and passive ROM exercises. The x-rays were done at 2 weeks, 4 weeks and 6 weeks follow up. All the complications were dealt with according to the protocols. The follow up was completed at 6 weeks postoperatively using Flynn's criteria as follows; ${ }^{6}$

\section{Flynn' Criteria}

\begin{tabular}{|l|c|c|}
\hline Rating & $\begin{array}{c}\text { Cosmetic factor } \\
\text { (Loss of carrying } \\
\text { angle in degrees) }\end{array}$ & $\begin{array}{c}\text { Functional factor } \\
\text { (Loss of } \\
\text { Movements } \\
\text { in degrees) }\end{array}$ \\
\hline Poor & $>15$ & $>15$ \\
\hline Fair & $11-15$ & $11-15$ \\
\hline Good & $6-10$ & $6-10$ \\
\hline Excellent & $0-5$ & $0-5$ \\
\hline
\end{tabular}

The data was analyzed using SPSS version 20. The demographic variables including age (5-12 years) and gender, were analyzed. Descriptive statistics like 
mean and standard deviation were used to present quantitative variables including age. Frequency distribution tables were used to present qualitative data including gender (male/ female) and Flynn's Criteria (excellent, good, fair, and poor) presented as frequency distribution tables. Confounding variables and effect modifiers were controlled by stratification of data. P-value $<0.05$ was be considered significant.

\section{Results}

This study was conducted on 254 patients of both sexes between the age of 5 years and 12 years. The results showed that the mean age of patients was $8.43 \pm 1.96$ years, where males have a mean age of $8.48 \pm 2.04$ years and females $8.38 \pm 1.90$ years. Among 254 patients, $119(46.9 \%)$ were male whereas, females were $135(53.1 \%)$. The results of this study showed that $202(79.5 \%)$ patients were fulfilling the satisfactory level of outcome, 52 (20.5\%) patient showed an unsatisfactory level of outcome according to operational definition (Table I), and 96 (80.6\%) males and $106(78.5 \%)$ females patients showed satisfactory outcome whereas $23(19.4 \%)$ males and 29 (21.5\%) female patients showed an unsatisfactory level of outcome, where p-value was 0.04 as in Table II. Out of 254 patients, 59 (23\%) showed excellent, $94(37 \%)$ good, 49 (19\%) fair and $52(20 \%)$ poor outcome as defined in operational definition. (Table III)

Table-I: Outcome of procedure among study subjects

\begin{tabular}{|l|c|c|c|}
\hline Outcome & Frequency & Percent & P-value \\
\hline Satisfactory & 202 & 79.5 & \multirow{2}{*}{0.04} \\
\cline { 1 - 3 } Unsatisfactory & 52 & 20.5 & \multirow{2}{*}{ Total } \\
\cline { 1 - 3 }
\end{tabular}

Table-II: Outcome of procedure among study subjects

\begin{tabular}{|l|c|c|c|}
\hline $\begin{array}{l}\text { Outcome of the } \\
\text { procedure }\end{array}$ & Males & Females & Total \\
\hline Satisfactory & $96(80.6 \%)$ & $106(78.5 \%)$ & $202(79.5 \%)$ \\
\hline Unsatisfactory & $23(19.4 \%)$ & $29(21.5 \%)$ & $52(20.5 \%)$ \\
\hline Total & $119(100 \%)$ & $135(100 \%)$ & $254(100 \%)$ \\
\hline
\end{tabular}

Table- III: Outcome according to Scoring system

\begin{tabular}{|l|c|c|c|}
\hline Outcome & Males & Female & Total \\
\hline Excellent & $31(26 \%)$ & $28(20.7 \%)$ & $59(23.22 \%)$ \\
\hline Good & $46(38.6 \%)$ & $48(33.5 \%)$ & $94(37 \%)$ \\
\hline Fair & $19(16 \%)$ & $30(22.2 \%)$ & $49(19.3 \%)$ \\
\hline Poor & $23(19.3 \%)$ & $29(21.5 \%)$ & $52(20.5 \%)$ \\
\hline Total & $119(100 \%)$ & $135(100 \%)$ & $254(100 \%)$ \\
\hline
\end{tabular}

In our series, no patient had any neurological injuries. Radial nerve deficit was found in 6 patients $(2.3 \%)$ before surgery, which recovered within 2 months after injury. In the present study, six patients (2.3\%) experienced an insignificant infection at the site of the insertion of wires that was managed by taking away the K-wire and giving oral antibiotics. Saucerization of the bone through lateral incision was employed in two patients $(0.8 \%)$ out of 254 who became infected with bone infection at the lateral cortex and did not improve with antibiotics given according to culture.

\section{Discussion}

Among all types of elbow fractures, 50\%-70\% consists of Supracondylar fractures of the humerus in children 3-10 years of age. ${ }^{7}$ The various suggested treatment techniques include closed reduction with plaster immobilization, traction followed by percutaneous pinning, and open reduction with internal fixation, but no single treatment method is considered ideal. ${ }^{8}$ To immobilize the fracture without pinning, one has to hyperflex the elbow which may compromise the vessels. Skin and skeletal traction are not the recommended methods of fracture stabilization. Open reduction and internal fixation with k-wires is hazardous due to soft tissue dissection in the presence of swelling and also has a high incidence of elbow stiffness and varus deformity. ${ }^{9}$

Nowadays method of choice is percutaneous pinning under image intensifier but controversies are present on the ideal positioning of Kirschner wires. As compared to open reduction, percutaneous pinning is cost-effective due to no use of sutures, lesser hospital stay, and no need for antibiotics. Percutaneous pinning as compared to cast immobilization has negligible chances of compartment syndrome and reduction loss. ${ }^{7}$ Danielson and Peterson observed loss of reduction while using a single wire pinning method. In previous studies, pinning with cross wires through medial and lateral epicondyles was the most commonly used method, with complication rates of 
about $2-8 \%$. Though this method of pinning is biomechanically stable but carries the risk of damaging the ulnar nerve. ${ }^{5,10}$ On the contrary, lateral pinning has fewer chances of nerve injury but is biomechanically not stable. ${ }^{8,10}$

Numerous studies conducted with amended fixation methods so that neurological insult (ulnar nerve) and fracture displacement is prevented. These show diversity in results regarding the possibility of instability, redo surgery due to displacement, nerve injuries, functional, and cosmesis. ${ }^{11,12}$ Dorgan's percutaneous k-wiring technique using the lateral side was used in our study. The crossed-wire alignment of wires, placing the wires through the radial side is analogous to that achieved by medial and lateral technique. In this technique, there is no danger of ulnar nerve injury. A literature search shows injury to the radial nerve while placing more proximal wire; however, which also can be bypassed by pinning a few degrees dorsal to the mid-coronal plane. According to Shannon's, ${ }^{13}$ study of 20 patients, there were no nerve injuries, the range of motion was comparable with the other side, and the mean carrying angle was $15^{\circ}$ (range $10^{\circ}-20^{\circ}$ ). Only k-wires associated complications were noted.

In our series, no patient had any neurological injuries. Radial nerve deficit was found in 6 patients $(2.3 \%)$ before surgery, which recovered within 2 months after injury. This was comparable with previous studies results, where no nerve palsy resulted. ${ }^{12,13}$ In the present study, six patients $(2.3 \%)$ experienced an insignificant infection at the site of insertion of wires that was managed by taking away the K-wire and giving oral antibiotics. Saucerization of the bone through lateral incision was employed in two patients $(0.8 \%)$ out of 254 who became infected with bone infection at the lateral cortex and did not improve with antibiotics given according to culture.

About 32 (12.6\%) patients expressed immoderate granulation tissue surrounding the wires but managed efficiently as daycare cases under antibiotics coverage with repeated dressings in OPD. Comparable outcomes were noted with Shannon study, ${ }^{13}$ where five patients $(40 \%)$ came out with extensive granulation tissue surrounding the wire.

\section{Conclusion}

Our study concluded that percutaneous pinning is an effective procedure for Gartland type 2 and type 3, supracondylar fractures of the humerus in the children, with a good outcome. There are fewer complications with this mode of treatment and less operative trauma and no problem of scar formation.

\section{Author Contribution}

MA: Interpretation of data and revising. KS: Interpretation of data. MKHM: Conception of work, design of work and revising. MSB \& GQK: Design of work, Acquisition and analysis of data and drafting. SR: Data Analysis, drafting and revising. All authors critically revised and approve its final version.

\section{Conflict of interest: None Resources of funding: Self}

\section{References}

1. Babal JC, Mehlman CT, Klein G. Nerve Injuries Associated with Pediatric Supracondylar Humeral Fractures: a Metaanalysis. J Pediatr Orthop 2010; 30: 253-3.

2. Grag B, Pankaj A, Malhotra R, Bhan S. Treatment of flexion type supracondylar humerus fracture in children. Journal of Orthopaedic Surgery. 2007; 15: 174-6.

3. Tiwari A, Kanoja RK, Kapoor SK. Surgical management for late presentation of supracondylar humeral fracture in children. Journal of Orthopaedic Surgery 2007; 15: 177-82.

4. Wael A, El-Said MA, Boghdady GW, Ali AS. Results of treatment of displaced supracondylar humeral fractures in children by percutaneous lateral cross-wiring technique. Strategies in Trauma and Limb Reconstruction 2008 Apr 1; 3(1): 1-7.

5. Maity A, Saha D, Roy DS. A prospective randomised, controlled clinical trial comparing medial and lateral entry pinning with lateral entry pinning for percutaneous fixation of displaced extension type supracondylar fractures of the humerus in children. Journal of Ortho Surg and Res 2012 Dec 1; 7(1): 6.

6. Iyengar SR, Hoffinger SA, Townsend DR. Early versus delayed reduction and pinning of type III displaced supracondylar fractures of the humerus in children: a comparative study. J Orthop Trauma 1999; 13(1): 51-5.

7. Shoaib M, Sultan S, Sahibzada SA, Ali A. Percutaneous pinning in displaced supracondylar fracture of humerus in children. Jour of Ayub Med Coll Abbottabad 2004; 16(4).

8. Devkota P, Khan JA, Acharya BM, Pradhan NM, Mainali LP, Singh M, et al. Outcome of supracondylar fractures of the humerus in children treated by closed reduction and percutaneous pinning. J Nepal Med Assoc 2008 Apr 1; 47(170): 66-70.

9. Cheng JC, Lam TP, Shen WY. Closed reduction and percutaneous pinning for type III displaced supracondylar fractures of the humerus in children. Jour of Ortho Trauma 1995; 9(6): 511-5. 
10. Brauer CA, Lee BM, Bae DS, Waters PM, Kocher MS. A systematic review of medial and lateral entry pinning versus lateral entry pinning for supracondylar fractures of the humerus. Jour of Ped Ortho 2007 Mar 1; 27(2): 181-6.

11. Sibinski M, Sharma H, Sherlock DA. Lateral versus crossed wire fixation for displaced extension supracondylar humeral fractures in children. Injury 2006 Oct 1;37(10): 961-5.
12. Kalenderer O, Reisoglu A, Surer L, Agus H. How should one treat iatrogenic ulnar injury after closed reduction and percutaneous pinning of paediatric supracondylar humeral fractures?. Injury 2008 Apr 1; 39(4): 463-6.

13. Shannon FJ, Mohan P, Chacko J, D'Souza LG. "Dorgan's" percutaneous lateral cross-wiring of supracondylar fractures of the humerus in children. Jour Ped Ortho 2004 Jul 1;24(4): 376-9. 